\title{
How Different is Wireless Access? Implications for Internet Mergers
}

\author{
Emanuele Giovannetti
}

\author{
January 2003
}

DAE Working Paper No. 0307

Not to be quoted without permission 


\begin{abstract}
Network hierarchies in the Internet are often not fixed: two providers can be simultaneously input supplier and retailer, in a routing process, while being horizontally competing in another. We introduce a stylised network model capturing these aspects of the Internet to study the impact of differentiation introduced by wireless access on prices and profits. We then study the incentives for, and welfare impact of, a merger between the wireless provider and a local bottleneck fixed access one. These effects crucially depend on the degree of differentiation between the wireless and fixed Internet access modalities. Pricing, at each router, follows the motor-way toll metaphor.
\end{abstract}

JEL Classification: L13, L86, L96,

Keywords: Mergers, Internet, Network Industries, Wireless Access, 


\title{
How Different is Wireless Access? Implications for Internet Mergers*
}

\author{
Emanuele Giovannetti ${ }^{\dagger}$ \\ Department of Applied Economics, \\ University of Cambridge.
}

January 2003

\section{Introduction}

By typing the key words: Internet, Wireless, Access a popular search engine returned 1,400,000 web pages containing these three terms; by refining the search adding the word Economics the number fell to the still astonishing 51,500. Together with the typical phenomenon of information overload, these numbers still indicate the relevance of wireless access to the Internet. By moving to Econlit we found no entries for Internet wireless access, 16 for Internet access, and 1017 for Internet. There is a clear discrepancy between the evolution of the Industry and the amount of research devoted to it in economics. The relevance of the wireless market is also attested to by the International Telecommunications Union forecast that in the year 2003 the world number of mobile users will overtake the number of fixed lines and by the initial diffusion of mobile standards for accessing the Internet such as the WAP (Wireless Application Protocol) and in the near future 3G mobile phones. The main characteristic of these new wireless standards is the increased differentiation they introduce in providing access to the Internet, because of the new set of wireless devices, their portability and operability. Also the introduction of wireless is based on volume pricing, as for the i-mode service introduced by NTT DoCoMo in Japan. All these issues are of particular relevance for the future of the $3 \mathrm{G}$ mobile standards.

*DAE, Austin Robinson Building, Sidgwick Avenue, Cambridge, CB3 9DE.

†The author acknowledges the support from the ESRC grant R000238563. He also wishes to thank the participants to the merger sessions of the ESEM 2002 Conference, and those of the CEPR Lisbon Workshop on the "Evolution of Network Industries" for their useful suggestions. I would also like to thank Frank Kelly, David Newbery and Tommaso Valletti for their useful advice at different stages of this paper. The usual disclaimer applies. 
This paper analyses, within a stylized model, the possible economic impact of wireless access on the Internet structure focussing on its price and ownership structure.

We focus on a stylized network topology to capture the competition between fixed and wireless access to the Internet in the presence of local fixed bottlenecks. The main questions we ask are: how will the increased differentiation introduced by wireless access affect the prices of the local bottleneck? Will retail and access prices increase or decrease due to increased differentiation? and finally what will the incentives and consequences be of a merger between the wireless provider and the fixed bottleneck?

We find that the answers to this questions depend on the trade off between consumers' preferences for variety in network access and the transaction costs due to multiple connections.

In particular we analyse a merger in one of the retail markets and, given the network topology introduced, this merger is horizontal for one of the markets but vertical for the other, it has therefore original effects typical of the connection-less structure of an I.P. (Internet Protocol) network such as the Internet. We find that for low differentiation the merger implies a closure of the wireless activity in the market where the merger is horizontal, maintaining however a reduced but still positive demand for wireless access in the other market, where the merger is vertical. For an even lower level of product differentiation there will be no incentive to merge. Low differentiation implies therefore drastic differences of the merger along the different dimensions of the network architecture. Finally with higher differentiation we will still observe different effects of the merger along its dimensions.

Preferences for variety, driving the results of the model, are likely to be affected by the appearance of new access standards in terms of extended portability as with the WAP or UMTS (Universal Mobile Telecommunications Systems) protocols. Extending the compatibility between access to an I.P. network and existing devices such as mobile phones, television sets or even wrist watches will indeed offer more variety and an increased willingness to combine sources of access.

After a stylized description of the linkages between Internet users and a brief review of some related literature, the remainder of the paper is organized as follows: in section 2 we describe the model: consumer utility, demand for sending rates and the effects of product differentiation on equilibrium prices and profits. Section 3 contains the main results of the paper, in it we analyse the effects of the merger on prices, profits and market structure. Finally section 4 contains the conclusions of the paper. All the proofs are contained in the Appendix. 


\subsection{Related literature}

The literature on Internet pricing has mainly focussed on congestion pricing ${ }^{1}$. One of the debated issues is whether prices should be charged only at the retail level $^{2}$ or if information packets should be priced at each hop along their path, following a motor-way toll metaphor. We adopt this last pricing structure to emphasize the non-fixed vertical relations between providers. Users will then pay a linear price for their sending rates. ${ }^{3}$

Strategic pricing problems in network industries have been analysed in detail in the economic literature ${ }^{4}$ mostly for traditional telephony networks where telephone companies originate and terminate calls to each other. These two-way networks models, see for example Armstrong [1] and Laffont, Rey and Tirole [11], are appropriate for telephone exchanges which take place along dedicated connections. In our paper we focus on a different network architecture aimed at capturing the connection-less features of I.P. networks. Instead of having two networks, each controlling an essential facility for the other (the access to its customers) we consider the case when the owner of the essential facility also competes against its downstream retailers for the routing of through-traffic.

\section{The Model}

Consider a network routing two different traffic flows: one connecting two locations, $B_{1}$ and $B_{2}$ and the other connecting $A_{1}$ to $A_{2}$. Each route linking the two locations passes through two local switches, (the four small boxes, in Figure 1 ), one at each end of the link. We assume that both $B_{1}$ and $A_{1}$ have a liberalized retail market for Internet access. Each market has two independent and competitive providers: in $B_{1}$ the competition is between ISP one which provides wireless access and ISP three which provides fixed access while in $A_{1}$ the wireless operator, ISP one, competes against the fixed operator: ISP two. Finally the destination locations have a different market structure: $B_{2}$, has a single monopolistic local provider, ISP two, while in $A_{2}$ ISP three terminates the information flows originated from $A_{1}$ by the mobile operator ISP one, while ISP two terminates the flows originated from $A_{1}$ by its retail router. The larger dotted boxes in Figure 1 show the ownership structure of the switches.

\footnotetext{
${ }^{1}$ For a collection of material on Internet congestion pricing see, for example, Richard Gibbens' web page [8] and for a seminal economic paper see MacKie-Mason and Varian [12].

${ }^{2}$ Schenker, Clark, Estrin and Herzog, [13] suggested using Edge Pricing, where the entire computation of the charges for the user is done at the access point so that an ISP charges the users of its network and then makes a sequence of bilateral agreements with adjacent providers.

${ }^{3}$ These could be downloads rates, from web hosting sites. This interpretation also explains better why traffic flows are one-way and not two-way ones.

${ }^{4}$ For an overview on the theory of access pricing and welfare considerations see Laffont and Tirole [10], Armstrong [2] and Estache and Valletti [7]
} 


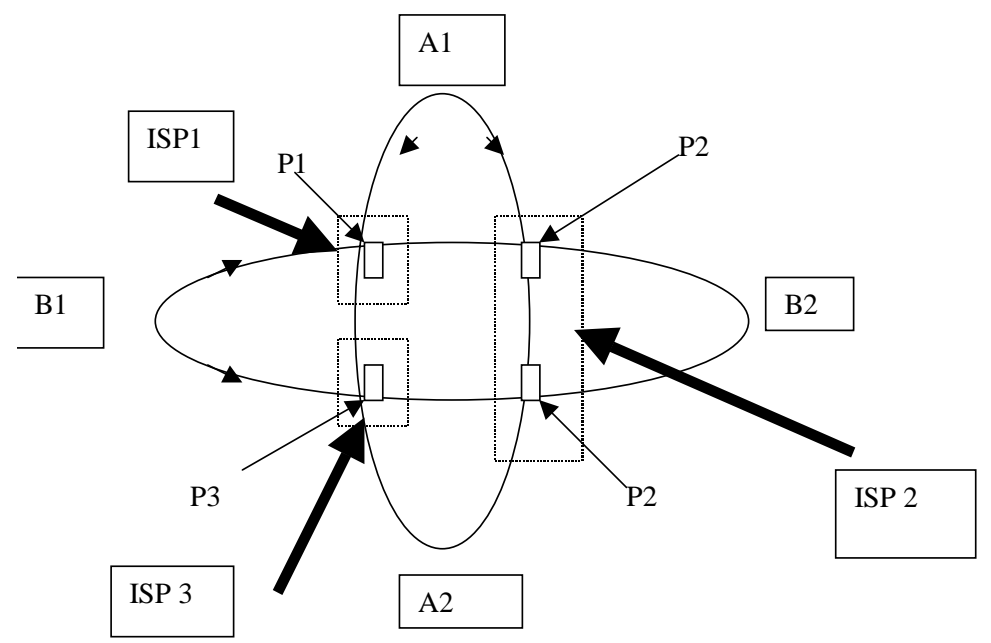

Figure 1

When users located in $B_{1}$ want to send information to $B_{2}$ they can choose any combination of traffic between the wireless operator, ISP one, and the fixed operator, ISP three, this is a typical example of multi-homing. The total sending rate from location $B_{1}$ to location $B_{2}$ is given by

$$
x^{B}=x_{1}^{B}+x_{3}^{B}
$$

where $x_{1}^{B}$ is the packet rate going through ISP one, with wireless access, and $x_{3}^{B}$ is the packet rate going through ISP three, the fixed access.

Similarly when users located in $A_{1}$ want to send information to $A_{2}$ they can choose any combination of traffic between the wireless operator, again ISP one, and the fixed operator, now, ISP two. The total sending rate from location $A_{1}$ to location $A_{2}$ is given by

$$
x^{A}=x_{1}^{A}+x_{2}^{A}
$$

We also assume that due to technological or regulatory reasons, no ISP is able to price discriminate between packets originating either in $A_{1}$ or in $B_{1}$ and therefore just charge one packet price whenever information is transmitted across its routers.

\subsection{Demand for sending rates}

The preferences of consumers located in $B_{1}$ over the composition of their sending rates through the mobile and the fixed operators are described by a quasi-linear utility function:

$$
U_{B}=\left[\alpha\left(x_{1}+x_{3}\right)-\left(\frac{1}{2}\left(x_{1}^{2}+x_{3}^{2}\right)\right)-\gamma\left(x_{1} x_{3}\right)\right]+m .
$$


and similarly for $A_{1}$ :

$$
U_{A}=\left[\alpha\left(x_{1}+x_{2}\right)-\left(\frac{1}{2}\left(x_{1}^{2}+x_{2}^{2}\right)\right)-\gamma\left(x_{1} x_{2}\right)\right]+m .
$$

The first term of the nonlinear part of equation (3), $\alpha\left(x_{1}+x_{3}\right)$, expresses the utility for the total sending rate weighted by the parameter $\alpha$ representing the size of the market. The second term, $-\frac{1}{2}\left(x_{1}^{2}+x_{3}^{2}\right)$, describes the consumers willingness to mix between different sources of access: fixed and wireless. This has an immediate interpretation: suppose we can access the network via a fixed connection or via a UMTS wireless connection; this variety of access modes allows the use of the most convenient connection at each moment. The third term of the utility function $(3),-\gamma\left(x_{1} x_{3}\right)$, accounts for the dis-utility derived from having two different providers. This dis-utility from variety expresses the opportunity costs for the time spent reading different contracts and bills, paying them separately, accessing two different support numbers for any query, learning different aspects of the connection and so on.

The parameter $\gamma$, weighting the relevance of these two terms of the utility function, describes the trade off between the desire for variety and the effort to minimize transaction and opportunity costs due to multi-homing. The more the services provided via wireless access differ from the fixed ones, the lower $\gamma$ will $\mathrm{be}^{5}$. When $\gamma$ tends to one there is no preference for variety: the transaction costs associated with having two different providers exactly offset the benefits derived from variety. Finally $m$, the linear part of (3) represents a composite, non Internet related, commodity.

Solving the consumer's problem given the utility function (3) we derive the market demands at location $B_{1}$ for sending rates through the wireless provider, ISP one, and through the fixed provider, ISP, three respectively:

$$
\begin{aligned}
& x_{1}^{B}=\frac{\alpha}{1+\gamma}-\frac{1}{1-\gamma^{2}}\left(p_{1}+p_{2}\right)+\frac{\gamma}{1-\gamma^{2}}\left(p_{3}+p_{2}\right) \\
& x_{3}^{B}=\frac{\alpha}{1+\gamma}-\frac{1}{1-\gamma^{2}}\left(p_{3}+p_{2}\right)+\frac{\gamma}{1-\gamma^{2}}\left(p_{1}+p_{2}\right)
\end{aligned}
$$

where: $\left(p_{1}+p_{2}\right)$ is the retail price for sending rates through the wireless ISP one, and $\left(p_{3}+p_{2}\right)$ the retail price of the fixed ISP three.

Similarly for the traffic originating at location $A_{1}$ we have:

\footnotetext{
${ }^{5}$ In the following we assume that $\gamma$ is always positive but less than one; this implies that the second term of the utility function, inducing a preference for variety is stronger than the third term, the costs of having two different providers.
} 


$$
\begin{aligned}
& x_{1}^{A}=\frac{\alpha}{1+\gamma}-\frac{1}{1-\gamma^{2}}\left(p_{1}+p_{3}\right)+\frac{\gamma}{1-\gamma^{2}}\left(2 p_{2}\right) \\
& x_{2}^{A}=\frac{\alpha}{1+\gamma}-\frac{1}{1-\gamma^{2}}\left(2 p_{2}\right)+\frac{\gamma}{1-\gamma^{2}}\left(p_{1}+p_{3}\right)
\end{aligned}
$$

These demand functions provide another interpretation for the parameter $\gamma$. Let $a=\frac{\alpha}{(1+\gamma)}, b=\frac{1}{1-\gamma^{2}}$, and $c=\frac{\gamma}{1-\gamma^{2}}$, the parameter $b$ represents the own price effect, or how a variation of a firm's own price will affect its market demand, and the term $c$, the cross price effect, expresses the effect on a firm's demand from a variation in its competitor's price. Hence the parameter $\gamma$, equal to the ratio of these two effects, $\gamma=\frac{c}{b}$, translates into an indicator of the degree of competition in the retail sector. When $\gamma$ equals zero, there is no competition effect and each provider has a safe market niche; when $\gamma$ tends to one the industry becomes perfectly competitive.

In the following we assume that the variable costs of routing a rate $x_{i}$ is zero at each network note, and that the fixed cost is zero, or sunk.

We assume that each provider sets its prices, simultaneously and without possibility to discriminate.

\subsubsection{Equilibrium prices and profits}

In the following proposition we study the effects of product differentiation on the equilibrium prices, wireless and fixed, market shares and profits for the model introduced above.

\section{Proposition 1}

a) The wireless price charged by ISP one, $p_{1}$, and the price charged by the fixed ISP three, $p_{3}$, are equal and both increasing in differentiation; the price charged by the fixed bottleneck, ISP two, $p_{2}$, is always lower than the other two prices and is also increasing in differentiation.

b) In the retail market in $A_{1}$, where the wireless provider competes against a vertically integrated fixed provider, the wireless market share:

$$
\frac{x_{1}^{A}}{x_{1}^{A}+x_{2}^{A}}=-\frac{1}{2} \frac{\gamma^{2}+2 \gamma+9}{\gamma^{2}-2 \gamma-11}
$$

is smaller than the fixed one and is decreasing in product differentiation

c) Providers' profits are decreasing in $\gamma$ and they tend to zero as $\gamma \rightarrow 1$.

Proof

See Appendix

\section{Discussion}


Although we introduce this case mainly for comparison purposes, proposition one is of interest on its own. The motor-way pricing structure used,(where a price is attached to each router traversed and the total price paid is given by the sum of these prices) removes some aspects of the vertical relations between routers: there is indeed no access price paid by the providers. However it is interesting to notice that in market $\mathrm{A}$, where the competition is between the vertically integrated ISP two and the non vertically integrated wireless ISP one, we observe asymmetric market shares. Moreover given the decreasing relation between wireless market share and product differentiation we find a complementarity between market power, due to vertical integration, and consumers' preferences for variety. Vertical integration matters the most when there is high differentiation, with lower differentiation the advantage of the vertically integrated competitor vanishes in the limit.

\section{Merger}

In this section we consider the incentives and the consequences of a merger between the fixed ISP two and the wireless ISP one. We focus on the effects of this merger on equilibrium prices, profits and market structure. Again these effects will depend on the degree of differentiation in the industry introduced by the wireless access.

\subsection{New Market structure}

Assume that the wireless ISP one and the fixed bottleneck ISP two have merged. As a consequence in market $A_{1}$ the same ISP will set both the wireless price, $p_{1}$, and the fixed one, $p_{2}$. This merger is, at the same time, an horizontal merger for market $A_{1}$, where the retail market is now controlled by one ISP and a vertical merger for the retail market in $B_{1}$. With respect to the traffic originated in $B_{1}$ the wireless provider, ISP one, is now vertically integrated with the upstream provider, ISP two, see figure below. 


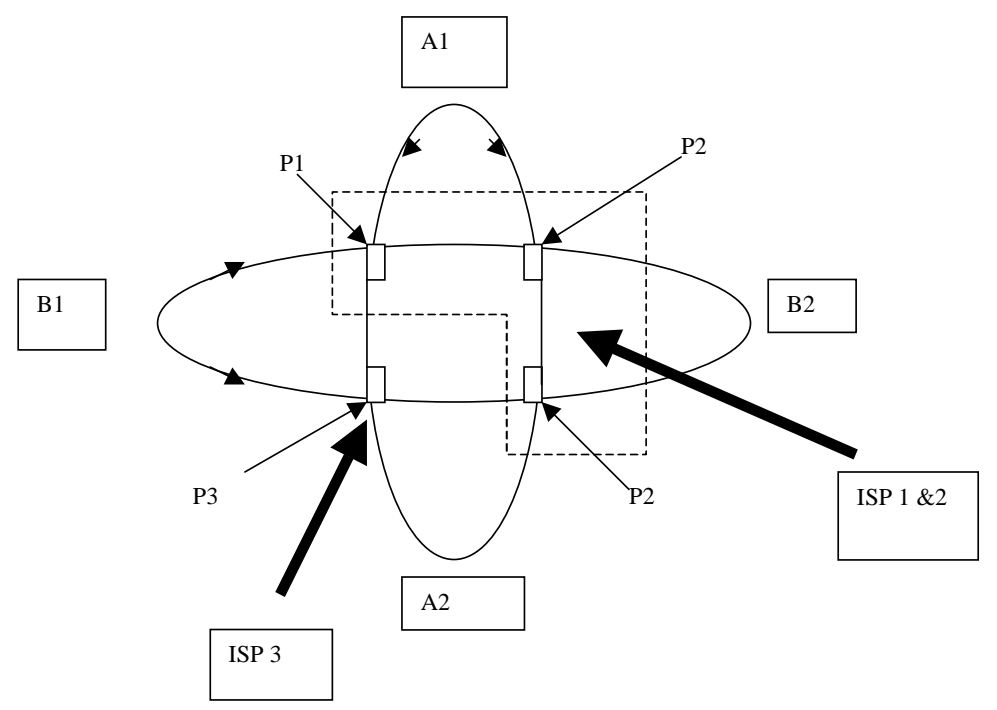

Figure 2

These combined effects of the merger on the network topology imply that the merger is neither horizontal nor vertical but has effects on both these dimensions whose interaction is analysed below.

In the next proposition we study the effects of the merger on the industry structure. These will be more relevant for the market where the merger is vertical, $B_{1}$, indeed with low differentiation, the merger will imply the closure of the retail market for the wireless provider in $B_{1}$.

\section{Proposition 2}

a) There is a critical value of the differentiation parameter: $\gamma$ such that: if

$$
\gamma \gtrsim 0.965
$$

then

$$
x_{1}^{B}=0
$$

b)

$$
\begin{gathered}
\gamma \lesssim 0.34 \rightarrow p_{3}>p_{1}>p_{2} \\
0.34 \lesssim \gamma \lesssim 0.55 \rightarrow p_{1}>p_{3}>p_{2}
\end{gathered}
$$

and finally

$$
\gamma \gtrsim 0.55 \rightarrow p_{1}>p_{2}>p 3
$$

\section{Proof}

See Appendix.

\section{Discussion}


This proposition deals with the effects of the merger on the prices. The most interesting thing is the presence of a threshold value for $\gamma$. For very low differentiation levels, for $\gamma$ above $\simeq 0.965$ the wireless price is such that demand on the $\mathrm{B}$ market equals zero, the same price is however low enough to maintain positive wireless demand on the $A$ market. This means that the strongest qualitative implication of the merger concerns the market where the merger is vertical and where there is still horizontal competition. Moreover part b of the proposition shows that with high differentiation wireless access price $\left(p_{1}\right)$ is cheaper than fixed access in the B market $\left(p_{3}\right)$, but more expensive than the fixed access in the A market $\left(p_{2}\right)$. With lower differentiation wireless access becomes more expensive than fixed access in both markets.

The next proposition focuses on the effects of the merger on the wireless market shares, and on prices in comparison with the pre-merger case.

\section{Proposition 3}

a) The wireless market shares for the $B$ market, is increased by the merger: iff

$$
\gamma \lesssim 0.34
$$

b) The wireless market share in the A market decreases iff

$$
0.34 \lesssim \gamma \lesssim 0.65
$$

Furthermore the merger will imply:

c) a reduction the retail price of the wireless provider, ISP one, $p_{1}$, for

$$
\gamma \lesssim 0.33
$$

d) a reduction of the price of the fixed provider ISP two, $p_{2}$, again for for

$$
\gamma \lesssim 0.33
$$

and

e) a reduction of the price of the fixed provider ISP three, $p_{3}$, only if

$$
0.33 \lesssim \gamma \lesssim 0.59
$$

\section{Proof}

See the Appendix.

\section{Discussion}

This proposition shows again the interaction between differentiation and merger effects. In fact for a highly differentiated industry the merger implies a higher wireless market share than in the pre-merger case(even thought with low differentiation its market shares equals zero!). The effects of differentiation on the 
market where the merger is horizontal is nonlinear. Indeed both a low and a high, but not an intermediate, differentiation levels imply an increase of the wireless market share in this market. Finally the merger has a positive effect in reducing all prices if there is high differentiation while it will only reduce the price of the excluded ISP and raising the others if there is low differentiation.

The graph below shows the difference between post-merger and pre-merger prices : the solid line for the wireless ISP one, while the dashed line and the dotted one show the same difference for ISP two and three respectively.

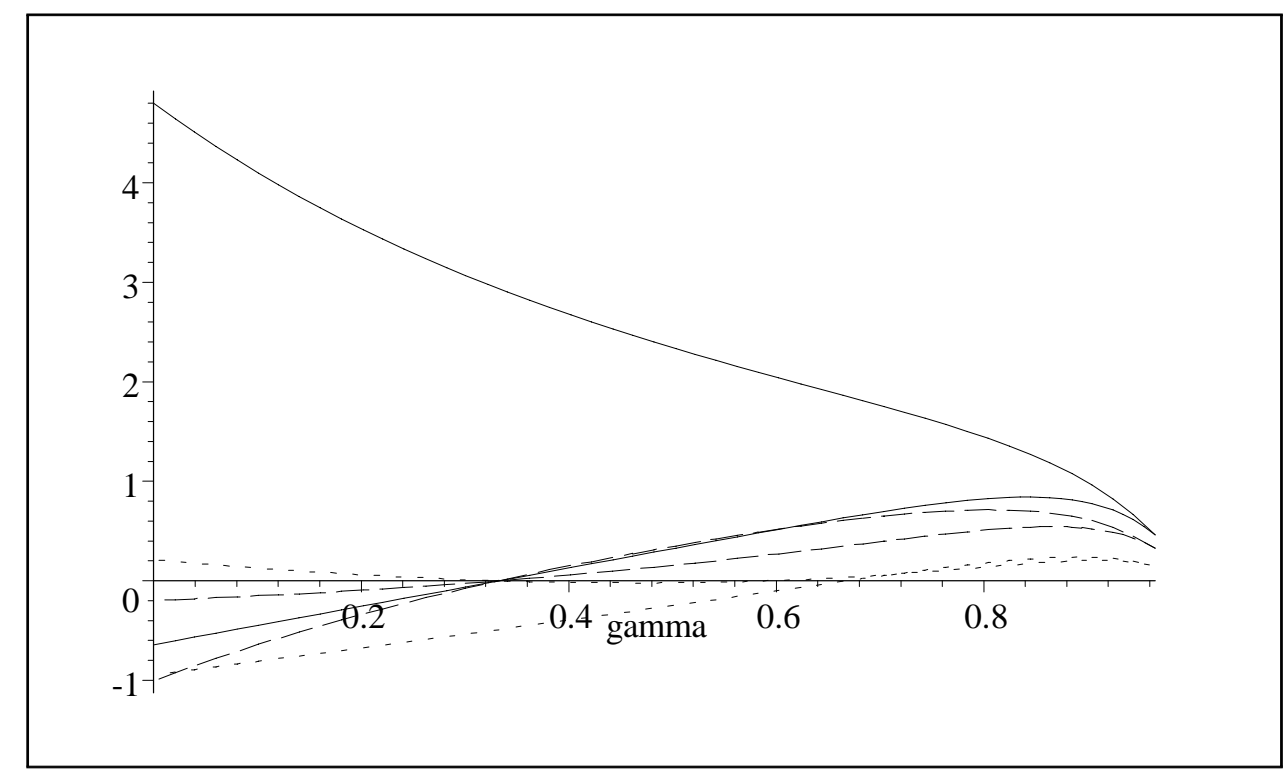

Fig. 3Post-merger Price Differential

\subsection{The incentives for mergers}

After having studied the consequences of a merger between the wireless ISP one and the fixed ISP two we are ready to analyse the incentives for these ISPs to merge and the profits impact of the merger on the third provider. In the next proposition we will see that there is a parameter range for which the merger can be profitable for all the providers, even for the ISP three, excluded from the merger.

\section{Proposition 4}

The merged firm ISP 1832 has no incentive to merge iff

$$
0.16 \lesssim \gamma \lesssim 0.32
$$

ISP three will loose profits from the merger, between ISP 1 and ISP 2, iff

$$
0.33 \lesssim \gamma \lesssim 0.58
$$

\section{Proof}




\section{See the Appendix}

\section{Discussion}

Clearly again the economic incentives for the merger depend on the degree of differentiation introduced by the wireless access. The merged firm gains from it if the industry is either very differentiated or if there is not too much differentiation. In a subset of this interval also the excluded firm gains from the merger. This suggest that the vertical merger of a direct retail competitor may indeed provide a collusive device to the industry as a whole.

Finally the next proposition explores the wider welfare effects of the merger; on the consumers of the separate markets, on them as a whole.

\section{Proposition 5}

The merger :

a) increases consumers' surplus in the B market iff

$$
\gamma \lesssim 0.318
$$

b) increases consumers' surplus in the A market iff

$$
\gamma \lesssim 0.335
$$

c) increases total consumer's surplus iff

$$
\gamma \lesssim 0.325
$$

d) decreases total profits iff

$$
0.333 \lesssim \gamma \lesssim 0.43
$$

and

e) increases total welfare iff

$$
\gamma \lesssim 0.325
$$

\section{Proof}

See the Appendix.

\section{Discussion}

In the graph below we have the consumers' surplus changes due to the merger, the solid line is for the $A_{1}$ market, and the dotted line for the $B_{1}$ market. From the graph we can see that on the $B_{1}$ market where the merger was vertical we have less intense effects than in the $A_{1}$ market, both when they are positive, with high differentiation and $\gamma \lesssim 0.318$ and when they are negative, with low differentiation and $\gamma \gtrsim 0.318$. 


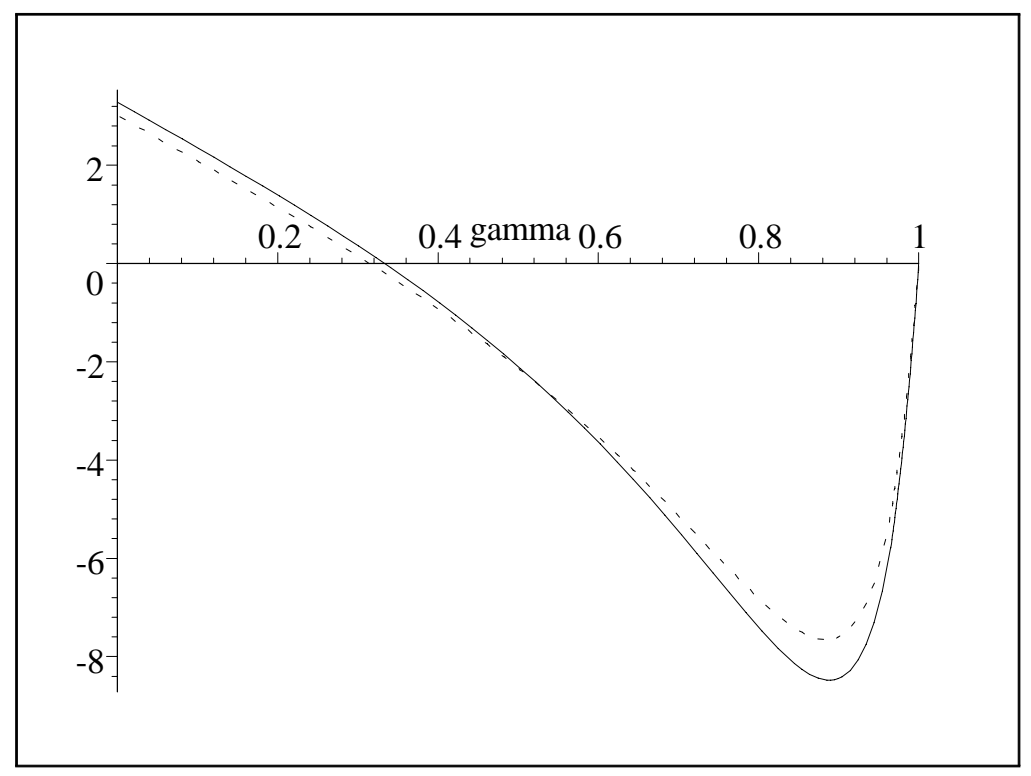

Fig. 4 Consumer welfare effect of the merger

\section{Conclusions}

In this paper we introduced a stylized representation of the Internet, where traffic flows are routed at different switches which charge a unit price independently of the initial and final destination of the data flow. We considered two interconnected competitive retail markets both supplying fixed and wireless access to the net, and two termination markets, one of which is monopolised by a single ISP, a bottleneck and is also vertically integrated with one of the retailers in one of the markets. These destination markets can be thought as web-hosting sites, so that the demand functions in the model refer to downloads demands.

Equilibrium prices and providers profits crucially depend on the degree of differentiation introduced in the industry by the wireless access. The more consumers appreciate the variety of the services introduced by wireless access the more the retail market is differentiated, the higher are prices for the operators and the smaller the market share for the wireless operator in the market where it competes against the vertically integrated fixed access provider. We then consider a merger in one of the retail markets, between the vertically integrated fixed access provider and the wireless one. This merger is horizontal for one of the markets but vertical for the other, it has therefore original effects typical of the connection-less structure of an I.P. network such as the Internet. The effects of the merger crucially depend on the degree of differentiation introduced by the wireless access. For low differentiation, the merger implies equilibrium prices which send the wireless demand for access to zero, in the market where the merger is vertical, maintaining however a positive demand for wireless access in the other market, where the merger is horizontal. Post merger prices are lower 
than pre-merger ones if there is low differentiation, they are higher otherwise. Consumer welfare diminishes as a result of the merger if there is not enough differentiation, while Industry profits will usually increase, apart for an intermediate differentiation parameter range, where they decrease.

Finally the interdependence of these effects is due to the assumption that providers are unable to price discriminate traffic of different origins, at their routers. With price discrimination the physical linkages of the Internet would not provide economic linkages across markets and no original analysis would be required.

\section{Appendix}

\section{Proof of Proposition 1}

Demands are given by

$$
\begin{aligned}
x_{1}^{B} & =\left(\frac{\alpha}{1+\gamma}-\frac{1}{1-\gamma^{2}}\left(p_{1}+p_{2}\right)+\frac{\gamma}{1-\gamma^{2}}\left(p_{3}+p_{2}\right)\right) \\
x_{1}^{A} & =\left(\frac{\alpha}{1+\gamma}-\frac{1}{1-\gamma^{2}}\left(p_{1}+p_{3}\right)+\frac{\gamma}{1-\gamma^{2}} 2 p_{2}\right) \\
x_{2}^{A} & =\left(\frac{\alpha}{1+\gamma}-\frac{1}{1-\gamma^{2}} 2 p_{2}+\frac{\gamma}{1-\gamma^{2}}\left(p_{1}+p_{3}\right)\right) \\
x_{3}^{B} & =\left(\frac{\alpha}{1+\gamma}-\frac{1}{1-\gamma^{2}}\left(p_{3}+p_{2}\right)+\frac{\gamma}{1-\gamma^{2}}\left(p_{1}+p_{2}\right)\right)
\end{aligned}
$$

where: $\left(p_{1}+p_{2}\right)$ is the retail price for sending rates through the wireless ISP one, and $\left(p_{3}+p_{2}\right)$ the retail price of the fixed ISP three.

To construct best reply functions we need to consider the possibility that a ISP sets prices as to "close" one market. This is relevant since firms now play with one price on two different markets and we need to verify if corner solutions are best replies. In the following we set up the Lagrangians for the three firms and we find that the internal solutions form an equilibrium for the game

\section{ISP one}

Imposing the non negativity constraints for the two final demands routed by ISP one, we have the Lagrangian function:

$$
\begin{aligned}
L_{1}= & \left(\begin{array}{c}
\left(\frac{\alpha}{1+\gamma}-\frac{1}{1-\gamma^{2}}\left(p_{1}+p_{2}\right)+\frac{\gamma}{1-\gamma^{2}}\left(p_{3}+p_{2}\right)\right)+ \\
\left(\frac{\alpha}{1+\gamma}-\frac{1}{1-\gamma^{2}}\left(p_{1}+p_{3}\right)+\frac{\gamma}{1-\gamma^{2}} 2 p_{2}\right)
\end{array}\right) p_{1}+ \\
& \mu_{1}\left(\frac{\alpha}{1+\gamma}-\frac{1}{1-\gamma^{2}}\left(p_{1}+p_{2}\right)+\frac{\gamma}{1-\gamma^{2}}\left(p_{3}+p_{2}\right)\right)+ \\
& \mu_{2}\left(\frac{\alpha}{1+\gamma}-\frac{1}{1-\gamma^{2}}\left(p_{1}+p_{3}\right)+\frac{\gamma}{1-\gamma^{2}} 2 p_{2}\right)
\end{aligned}
$$


from which we can derive the reaction function for ISP one:

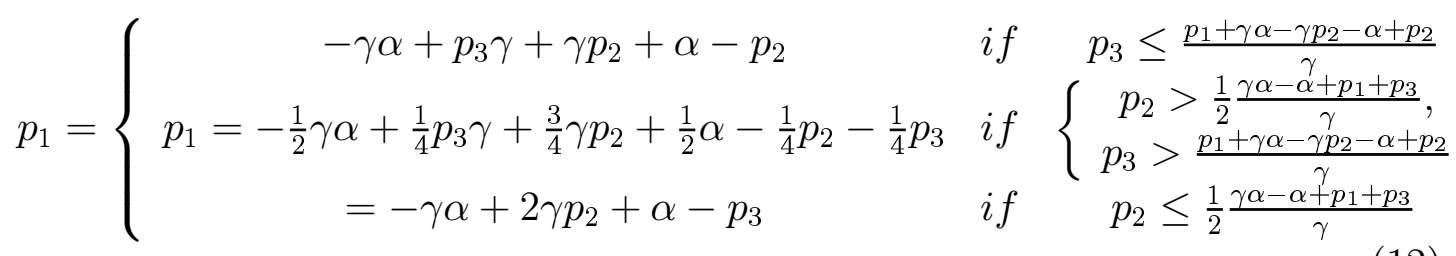

ISP two The Lagrangian for ISP two is given by

$$
\begin{aligned}
L_{2}= & p_{2}\left(\begin{array}{c}
\left(\frac{\alpha}{1+\gamma}-\frac{1}{1-\gamma^{2}}\left(p_{1}+p_{2}\right)+\frac{\gamma}{1-\gamma^{2}}\left(p_{3}+p_{2}\right)\right)+ \\
\left(\frac{\alpha}{1+\gamma}-\frac{1}{1-\gamma^{2}}\left(p_{3}+p_{2}\right)+\frac{\gamma}{1-\gamma^{2}}\left(p_{1}+p_{2}\right)\right)
\end{array}\right) \\
& +2 p_{2}\left(\frac{\alpha}{1+\gamma}-\frac{1}{1-\gamma^{2}} 2 p_{2}+\frac{\gamma}{1-\gamma^{2}}\left(p_{1}+p_{3}\right)\right) \\
& +\lambda\left(\frac{\alpha}{1+\gamma}-\frac{1}{1-\gamma^{2}} 2 p_{2}+\frac{\gamma}{1-\gamma^{2}}\left(p_{1}+p_{3}\right)\right)
\end{aligned}
$$

and its reaction function:

$$
p_{2}=\left\{\begin{array}{ccc}
-\frac{1}{2} \gamma \alpha+\frac{1}{2} p_{1} \gamma+\frac{1}{2} p_{3} \gamma+\frac{1}{2} \alpha & \text { if } & p_{1} \leq \frac{\gamma \alpha-p_{3} \gamma-\alpha+2 p_{2}}{\gamma} \\
p_{2}=-\frac{1}{4} \frac{3 p_{3} \gamma+3 p_{1} \gamma-4 \gamma \alpha-p_{1}-p_{3}+4 \alpha}{\gamma-3} & \text { if } & p_{1}>\frac{\gamma \alpha-p_{3} \gamma-\alpha+2 p_{2}}{\gamma}
\end{array}\right.
$$

ISP three The Lagrangian is given by:

$$
\begin{aligned}
L_{3}= & \left(\begin{array}{c}
\left(\frac{\alpha}{1+\gamma}-\frac{1}{1-\gamma^{2}}\left(p_{3}+p_{2}\right)+\frac{\gamma}{1-\gamma^{2}}\left(p_{1}+p_{2}\right)\right) \\
+\left(\frac{\alpha}{1+\gamma}-\frac{1}{1-\gamma^{2}}\left(p_{1}+p_{3}\right)+\frac{\gamma}{1-\gamma^{2}} 2 p_{2}\right)
\end{array}\right) p_{3} \\
& +\varphi\left(\frac{\alpha}{1+\gamma}-\frac{1}{1-\gamma^{2}}\left(p_{3}+p_{2}\right)+\frac{\gamma}{1-\gamma^{2}}\left(p_{1}+p_{2}\right)\right)
\end{aligned}
$$

and its reaction function:

$$
p_{3}=\left\{\begin{array}{ccc}
-\gamma \alpha+p_{1} \gamma+\gamma p_{2}+\alpha-p_{2} & \text { if } & p_{1} \leq-\frac{-\gamma \alpha+\gamma p_{2}+\alpha-p_{3}-p_{2}}{\gamma} \\
-\frac{1}{2} \gamma \alpha+\frac{1}{4} p_{1} \gamma+\frac{3}{4} \gamma p_{2}+\frac{1}{2} \alpha-\frac{1}{4} p_{2}-\frac{1}{4} p_{1} & \text { if } & p_{1}>-\frac{-\gamma \alpha+\gamma p_{2}+\alpha-p_{3}-p_{2}}{\gamma}
\end{array}\right.
$$


The internal solutions of the system of reaction functions (12), (14) and (16) are given by:

$$
\begin{aligned}
& p_{1}=2 \alpha \frac{\gamma^{2}+4 \gamma-5}{7 \gamma^{2}+10 \gamma-29} \\
& p_{2}=4\left(\gamma^{2}+\gamma-2\right) \frac{\alpha}{7 \gamma^{2}+10 \gamma-29} \\
& p_{3}=2 \alpha \frac{\gamma^{2}+4 \gamma-5}{7 \gamma^{2}+10 \gamma-29}
\end{aligned}
$$

Before concluding that these are equilibrium prices we need to check whether, at these prices, any constraint is binding.

By substituting (17) into (10) we obtain the actual equilibrium demands

$$
\begin{aligned}
x_{1}^{B} & =\alpha \frac{\gamma^{2}-2 \gamma-11}{\left(7 \gamma^{2}+10 \gamma-29\right)(1+\gamma)} \\
x_{1}^{A} & =-\alpha \frac{\gamma^{2}+2 \gamma+9}{\left(7 \gamma^{2}+10 \gamma-29\right)(1+\gamma)} \\
x_{2}^{A} & =\alpha \frac{3 \gamma^{2}-2 \gamma-13}{\left(7 \gamma^{2}+10 \gamma-29\right)(1+\gamma)} \\
x_{3}^{B} & =\alpha \frac{\gamma^{2}-2 \gamma-11}{\left(7 \gamma^{2}+10 \gamma-29\right)(1+\gamma)}
\end{aligned}
$$

which are always positive for $\gamma \in(0,1)$.

a) is easily derived from(17).

b) From (18) one obtains market shares for the asymmetric market A

$$
\frac{x_{1}^{A}}{x_{1}^{A}+x_{2}^{A}}=-\frac{1}{2} \frac{\gamma^{2}+2 \gamma+9}{\gamma^{2}-2 \gamma-11}
$$

which is clearly increasing in $\gamma$.

c) Equilibrium profits:

From (17) (18)we obtain:the equilibrium profits for the three operators

$$
\begin{aligned}
& \pi_{1}=-8(\gamma+5)\left(\gamma^{2}+4 \gamma-5\right) \frac{\alpha^{2}}{(1+\gamma)\left(7 \gamma^{2}+10 \gamma-29\right)^{2}} \\
& \pi_{2}=32\left(\gamma^{2}+\gamma-2\right)\left(\gamma^{2}-\gamma-6\right) \frac{\alpha^{2}}{(1+\gamma)\left(7 \gamma^{2}+10 \gamma-29\right)^{2}} \\
& \pi_{3}=-8(\gamma+5)\left(\gamma^{2}+4 \gamma-5\right) \frac{\alpha^{2}}{(1+\gamma)\left(7 \gamma^{2}+10 \gamma-29\right)^{2}}
\end{aligned}
$$

By differentiating (19) with respect to $\gamma$ it is easily seen there these are all decreasing in $\gamma$ and they reach zero as $\gamma \rightarrow 1$. 


\section{Proof of Preposition 2}

To construct best reply functions after the merger we need again to consider the possibility that a ISP sets prices as to "close" one market. In the following we set up the Lagrangians for the two firms and we analyse the conditions under which the internal solutions form an equilibrium for the game.

ISP $1 \& 2$

The Lagrangian for the merged ISP $1 \& 2$ is given by:

$$
\begin{aligned}
L_{1 \& 2}= & \left(\begin{array}{c}
\left(\frac{\alpha}{1+\gamma}-\frac{1}{1-\gamma^{2}}\left(p_{1}+p_{2}\right)+\frac{\gamma}{1-\gamma^{2}}\left(p_{3}+p_{2}\right)\right)+ \\
\left(\frac{\alpha}{1+\gamma}-\frac{1}{1-\gamma^{2}}\left(p_{1}+p_{3}\right)+\frac{\gamma}{1-\gamma^{2}} 2 p_{2}\right)
\end{array}\right) p_{1} \\
& +p_{2}\left(\begin{array}{c}
\left(\frac{\alpha}{1+\gamma}-\frac{1}{1-\gamma^{2}}\left(p_{1}+p_{2}\right)+\frac{\gamma}{1-\gamma^{2}}\left(p_{3}+p_{2}\right)\right)+ \\
\left(\frac{\alpha}{1+\gamma}-\frac{1}{1-\gamma^{2}}\left(p_{3}+p_{2}\right)+\frac{\gamma}{1-\gamma^{2}}\left(p_{1}+p_{2}\right)\right)
\end{array}\right) \\
& +2 p_{2}\left(\frac{\alpha}{1+\gamma}-\frac{1}{1-\gamma^{2}} 2 p_{2}+\frac{\gamma}{1-\gamma^{2}}\left(p_{1}+p_{3}\right)\right)+ \\
& \mu_{1}\left(\frac{\alpha}{1+\gamma}-\frac{1}{1-\gamma^{2}}\left(p_{1}+p_{2}\right)+\frac{\gamma}{1-\gamma^{2}}\left(p_{3}+p_{2}\right)\right)+ \\
& +\mu_{2}\left(\frac{\alpha}{1+\gamma}-\frac{1}{1-\gamma^{2}}\left(p_{1}+p_{3}\right)+\frac{\gamma}{1-\gamma^{2}} 2 p_{2}\right)+ \\
& \lambda\left(\frac{\alpha}{1+\gamma}-\frac{1}{1-\gamma^{2}} 2 p_{2}+\frac{\gamma}{1-\gamma^{2}}\left(p_{1}+p_{3}\right)\right)
\end{aligned}
$$

from which we derive the reaction functions:

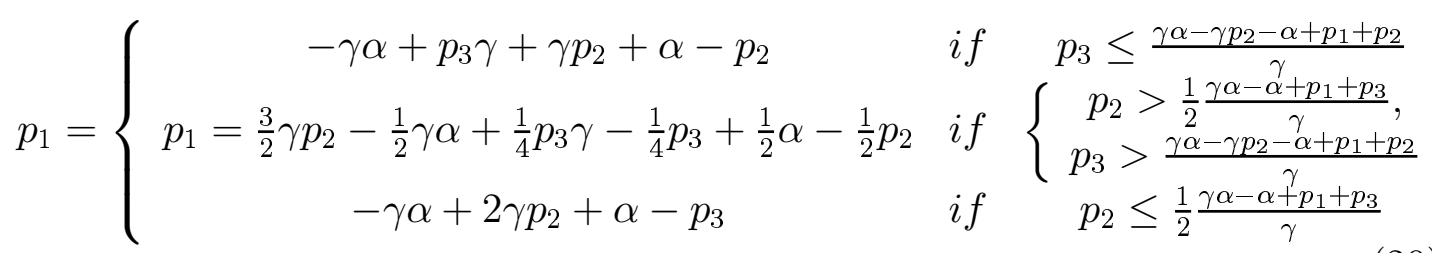

and

$$
p_{2}=\left\{\begin{array}{ccc}
-\frac{1}{2} \gamma \alpha+\frac{1}{2} p_{1} \gamma+\frac{1}{2} p_{3} \gamma+\frac{1}{2} \alpha & \text { if } & p_{1} \leq \frac{\gamma \alpha-p_{3} \gamma-\alpha+2 p_{2}}{\gamma} \\
-\frac{1}{4} \frac{6 p_{1} \gamma-4 \gamma \alpha+3 p_{3} \gamma+4 \alpha-p_{3}-2 p_{1}}{\gamma-3} & \text { if } & p_{1}>\frac{\gamma \alpha-p_{3} \gamma-\alpha+2 p_{2}}{\gamma}
\end{array}\right.
$$

For ISP three we have:

$$
\begin{aligned}
L_{3}= & \left(\begin{array}{c}
\left(\frac{\alpha}{1+\gamma}-\frac{1}{1-\gamma^{2}}\left(p_{3}+p_{2}\right)+\frac{\gamma}{1-\gamma^{2}}\left(p_{1}+p_{2}\right)\right)+ \\
\left(\frac{\alpha}{1+\gamma}-\frac{1}{1-\gamma^{2}}\left(p_{1}+p_{3}\right)+\frac{\gamma}{1-\gamma^{2}} 2 p_{2}\right)
\end{array}\right) p_{3} \\
& +\varphi_{1}\left(\frac{\alpha}{1+\gamma}-\frac{1}{1-\gamma^{2}}\left(p_{3}+p_{2}\right)+\frac{\gamma}{1-\gamma^{2}}\left(p_{1}+p_{2}\right)\right)
\end{aligned}
$$


and its reaction function

$p_{3}=\left\{\begin{array}{cll}-\gamma \alpha+p_{1} \gamma+\gamma p_{2}+\alpha-p_{2} & \text { if } & p_{1} \leq \frac{\gamma \alpha-p_{2} \gamma-\alpha+p_{3}+p_{2}}{\gamma} \\ p_{3}=-\frac{1}{2} \gamma \alpha+\frac{1}{4} p_{1} \gamma+\frac{3}{4} \gamma p_{2}+\frac{1}{2} \alpha-\frac{1}{4} p_{2}-\frac{1}{4} p_{1} & \text { if } & p_{1}>\frac{\gamma \alpha-p_{2} \gamma-\alpha+p_{3}+p_{2}}{\gamma}\end{array}\right.$

From $(20),(21)$ and (22)we obtain the candidate ${ }^{6}$ post-merger equilibrium prices:

$$
\begin{aligned}
& p_{1}=\left(\frac{1}{2}\left(11 \gamma^{2}+12 \gamma-23\right) \frac{\alpha}{8 \gamma^{2}+27 \gamma-41}\right) \\
& p_{2}=\left(\frac{7}{2} \alpha \frac{2 \gamma+\gamma^{2}-3}{8 \gamma^{2}+27 \gamma-41}\right) \\
& p_{3}=\left(-5 \alpha \frac{\gamma^{2}-4 \gamma+3}{8 \gamma^{2}+27 \gamma-41}\right)
\end{aligned}
$$

We need however to check if the constraints are binding at these candidate equilibrium prices. We start by checking whether (23) satisfy the constraints in the best reply functions: (20), (21) and (22).

We start from the wireless price $p_{1}$ and check the constraints given by (20) .

$$
p_{3}>\frac{\gamma \alpha-\gamma p_{2}-\alpha+p_{1}+p_{2}}{\gamma} \rightarrow p_{1}<-\gamma \alpha+p_{3} \gamma+p_{2} \gamma+\alpha-p_{2}
$$

By substituting (23) into (24) we have:

$$
\frac{1}{2} \alpha \frac{2 \gamma^{2}-59 \gamma+38+19 \gamma^{3}}{8 \gamma^{2}+27 \gamma-41}<0
$$

this is not satisfied for $\gamma \gtrsim 0.965$. For these values of $\gamma$ the merged firm will therefore choose a corner solution for setting the price $p_{1}$. for the market in $B_{1}$.

It is easy to show that (24) is the only constraint that is not satisfied at the prices (23) in the three best reply functions

\section{Corner Solutions}

From the previous analysis we have see that for $\gamma \gtrsim 0.965$ the price $p_{1}$ is chosen to close the market in $B$. It is given by the corner solution. $\mathrm{f}$ In the following we need to verify the new equilibrium prices given the corner best reply for $p_{1}$.

$$
p_{1}=-\gamma \alpha+p_{3} \gamma+p_{2} \gamma+\alpha-p_{2}
$$

The new best reply system where $p_{1}$ is chosen from the corner solution while $p_{2}$ and $p_{3}$ are still chosen from the internal best replies will be:

\footnotetext{
${ }^{6}$ These are only equilibria if there are not binding constraints. We'll see shortly that this is not always the case.
} 


$$
\begin{gathered}
p_{1}=-\gamma \alpha+p_{3} \gamma+p_{2} \gamma+\alpha-p_{2} \\
p_{2}=-\frac{1}{4} \frac{6 p_{1} \gamma-4 \gamma \alpha+3 p_{3} \gamma+4 \alpha-p_{3}-2 p_{1}}{\gamma-3} \\
p_{3}=-\frac{1}{2} \gamma \alpha+\frac{1}{4} p_{1} \gamma+\frac{3}{4} \gamma p_{2}+\frac{1}{2} \alpha-\frac{1}{4} p_{2}-\frac{1}{4} p_{1}
\end{gathered}
$$

providing us with new corner equilibrium prices:

$$
\begin{aligned}
& p_{1}=\left(\gamma^{3}+17 \gamma^{2}+15 \gamma-33\right) \frac{\alpha}{-40-27 \gamma+30 \gamma^{2}+17 \gamma^{3}} \\
& p_{2}=\alpha \frac{15 \gamma^{2}-7+11 \gamma^{3}-19 \gamma}{-40-27 \gamma+30 \gamma^{2}+17 \gamma^{3}} \\
& p_{3}=2\left(3 \gamma^{2}+2 \gamma-5\right) \frac{\alpha}{17 \gamma^{2}+13 \gamma-40}
\end{aligned}
$$

We now need to verify whether substituting (25) into to the $p_{2}$ component of the ISP1\&2 best replies, (21) and into ISP three one (22) these conditions will remain satisfied or not.

We start from ISP 2: the demand $x_{2}^{A}$, at the new prices given by (25)remains positive.

The same applies for Isp 3 demand $x_{3}^{B}$ at the new prices (25).

This proves that when ISP $1 \& 2$ chooses the price $p_{1}$ from its corner best reply and shuts the wireless market in $B_{1}$, while maintaining the wireless open for $A_{1}$ market all the other prices: $p_{2}$, and $p_{3}$, are still derived from internal best replies. This implies that after the post-merger corner the equilibrium prices are given by (25) for $\gamma \gtrsim 0.965$.

b) An analysis of (25) shows clearly this part of the proposition.

\section{Proof of proposition 3}

a) and b) From the corner equilibrium prices given by equation (25) and from the internal equilibrium ones given by equation (23), we obtain the market share of the wireless provider ISP one in location $B_{1}$ :

$$
\frac{x_{1}^{B}}{x_{1}^{B}+x_{3}^{B}}=\left\{\begin{array}{cll}
\frac{19 \gamma^{2}+21 \gamma-38}{17 \gamma^{2}+28 \gamma-69} & \text { if } & \gamma \lesssim 0.965 \\
0 & \text { if } & \gamma>0.965
\end{array}\right.
$$

and the market share of the wireless provider ISP one at location $A_{1}$ :

$$
\frac{x_{1}^{A}}{x_{1}^{A}+x_{2}^{A}}=\left\{\begin{array}{lll}
\frac{2 \gamma^{2}+13 \gamma-29}{17 \gamma^{2}+28 \gamma-69} & \text { if } & \gamma \lesssim 0.965 \\
-\frac{5 \gamma^{2}+10 \gamma-3}{5 \gamma^{2}-2 \gamma-23} & \text { if } & \gamma>0.965
\end{array}\right.
$$

The effect of the merger on the market shares is obtained by difference with the pre-merger market shares expressions: 


$$
\Delta \frac{x_{1}^{A}}{x_{1}^{A}+x_{2}^{A}}=\left\{\begin{array}{cll}
\frac{1}{2} \frac{21 \gamma^{4}+80 \gamma^{3}-14 \gamma^{2}-56 \gamma+17}{\left(17 \gamma^{2}+28 \gamma-69\right)\left(\gamma^{2}-2 \gamma-11\right)} & \text { if } & \gamma \lesssim 0.965 \\
-\frac{1}{2} \frac{5 \gamma^{4}-174 \gamma^{2}-144 \gamma+273-8 \gamma^{3}}{\left(5 \gamma^{2}-2 \gamma-23\right)\left(\gamma^{2}-2 \gamma-11\right)} & \text { if } & \gamma>0.965
\end{array}\right.
$$

and

$$
\Delta \frac{x_{1}^{B}}{x_{1}^{B}+x_{3}^{B}}=\left\{\begin{array}{ccc}
\frac{7}{2} \frac{3 \gamma^{2}+2 \gamma-1}{17 \gamma^{2}+28 \gamma-69} & \text { if } & \gamma \lesssim 0.965 \\
-\frac{1}{2} & \text { if } & \gamma>0.965
\end{array}\right.
$$

c), d) and e) The post-merger internal equilibrium prices, when $\gamma \lesssim 0.965$, are given by (23),

the post merger corner prices are given by (25) and the pre-merger ones are given by (17). By subtracting (17) from (23)we obtain the price effect of the merger for $\gamma \lesssim 0.965$ and we have internal equilibrium prices:

$$
\begin{aligned}
\Delta p_{1 i n t} & =\frac{3}{2} \alpha \frac{15 \gamma^{4}-14 \gamma^{3}-156 \gamma^{2}+206 \gamma-51}{\left(8 \gamma^{2}+27 \gamma-41\right)\left(7 \gamma^{2}+10 \gamma-29\right)} \\
\Delta p_{2 i n t} & =-\frac{1}{2} \alpha \frac{112 \gamma^{3}-30 \gamma^{2}-144 \gamma+15 \gamma^{4}+47}{\left(8 \gamma^{2}+27 \gamma-41\right)\left(7 \gamma^{2}+10 \gamma-29\right)} \\
\Delta p_{3 i n t} & =-\alpha \frac{51 \gamma^{4}+28 \gamma^{3}-186 \gamma^{2}+132 \gamma-25}{\left(8 \gamma^{2}+27 \gamma-41\right)\left(7 \gamma^{2}+10 \gamma-29\right)}
\end{aligned}
$$

By subtracting (17) from (25)we obtain the price effect of the merger for $\gamma \gtrsim$ 0.965:

$$
\begin{aligned}
\Delta p_{1 \mathrm{corn}} & =-\alpha \frac{27 \gamma^{5}+67 \gamma^{4}-230 \gamma^{3}-22 \gamma^{2}+715 \gamma-557}{\left(-40-27 \gamma+30 \gamma^{2}+17 \gamma^{3}\right)\left(7 \gamma^{2}+10 \gamma-29\right)} \\
\Delta p_{21 \text { corn }} & =\alpha \frac{27 \gamma^{4}-178 \gamma^{3}-166 \gamma^{2}+425 \gamma-117+9 \gamma^{5}}{\left(-40-27 \gamma+30 \gamma^{2}+17 \gamma^{3}\right)\left(7 \gamma^{2}+10 \gamma-29\right)} \\
\Delta p_{3 \mathrm{corn}} & =2 \alpha \frac{4 \gamma^{4}-37 \gamma^{3}-29 \gamma^{2}+117 \gamma-55}{\left(17 \gamma^{2}+13 \gamma-40\right)\left(7 \gamma^{2}+10 \gamma-29\right)}
\end{aligned}
$$

c) d) and e) are clearly derived from (27) and (28).

\section{Proof of proposition 4}

From (23)we obtain the post merger profits when $\gamma \lesssim 0.965$ :

$$
\pi_{1 \& 2}=\frac{5}{2} \alpha^{2} \frac{956 \gamma-286 \gamma^{2}+113 \gamma^{4}-372 \gamma^{3}+56 \gamma^{5}-467}{\left(8 \gamma^{2}+27 \gamma-41\right)^{2}\left(-1+\gamma^{2}\right)}
$$

and

$$
\pi_{3}=-50(\gamma-3)\left(\gamma^{2}-4 \gamma+3\right) \frac{\alpha^{2}}{(1+\gamma)\left(8 \gamma^{2}+27 \gamma-41\right)^{2}}
$$



by

By using (25) we will have that post-merger profits when $\gamma \gtrsim 0.965$ are given

$$
\begin{gathered}
\pi_{1 \& 2}=2 \alpha^{2} \frac{135 \gamma^{5}+217 \gamma^{4}-666 \gamma^{3}-750 \gamma^{2}+851 \gamma+213}{\left(17 \gamma^{2}+13 \gamma-40\right)^{2}(1+\gamma)^{2}} \\
\pi_{3}=-8(3 \gamma+5)\left(3 \gamma^{2}+2 \gamma-5\right) \frac{\alpha^{2}}{\left(17 \gamma^{2}+13 \gamma-40\right)\left(-40-27 \gamma+30 \gamma^{2}+17 \gamma^{3}\right)}
\end{gathered}
$$

By subtracting the pre-merger profits from the post merger ones we can derive the merger incentives for the parameter range where internal solutions are equilibria, (the high differentiation case).

By subtracting(19) from (29) and (30)

we have

$$
\begin{aligned}
\Delta \pi_{1 \& 2}^{h d}= & \frac{1}{2}\left(\begin{array}{c}
9624 \gamma^{8}+53981 \gamma^{7}+31205 \gamma^{6}-197227 \gamma^{5}- \\
122843 \gamma^{4}+338863 \gamma^{3}-123113 \gamma^{2}+9183 \gamma+327
\end{array}\right) * \\
& \left(\frac{\alpha^{2}}{\left(7 \gamma^{2}+10 \gamma-29\right)^{2}(1+\gamma)\left(8 \gamma^{2}+27 \gamma-41\right)^{2}}\right)
\end{aligned}
$$

and

$$
\Delta \pi_{3}^{h d}=-2 \alpha^{2} \frac{\left(\begin{array}{c}
969 \gamma^{7}-9107 \gamma^{6}-33459 \gamma^{5}+67233 \gamma^{4}+ \\
88075 \gamma^{3}-216201 \gamma^{2}+123615 \gamma-21125
\end{array}\right)}{\left(7 \gamma^{2}+10 \gamma-29\right)^{2}(1+\gamma)\left(8 \gamma^{2}+27 \gamma-41\right)^{2}}
$$

By subtracting the sum of the pre-merger profits of ISP one and two (19) from (31) we obtain the incentive for the merger for ISP one and two under low differentiation and corner prices:

$$
\Delta \pi_{1 \& 2}^{l d}=2 \alpha^{2} \frac{\left(\begin{array}{c}
-306428 \gamma^{3}+196586 \gamma^{4}-18278 \gamma^{5}-180616 \gamma^{2}+627031 \gamma+ \\
1991 \gamma^{9}+18993 \gamma^{8}+23364 \gamma^{7}-74576 \gamma^{6}-288067
\end{array}\right)}{\left(17 \gamma^{2}+13 \gamma-40\right)^{2}(1+\gamma)^{2}\left(7 \gamma^{2}+10 \gamma-29\right)^{2}}
$$

while subtracting from (32) the pre-merger profits of ISP three (19) we obtain the profit effect for ISP three of the merger for ISP one and two under low differentiation and corner prices:

$$
\begin{aligned}
\Delta \pi_{3}^{l d}= & -8\left(\begin{array}{c}
152 \gamma^{7}-754 \gamma^{6}-7181 \gamma^{5}-1217 \gamma^{4}+ \\
30094 \gamma^{3}-364 \gamma^{2}-39705 \gamma+18975
\end{array}\right) * \\
& \left(\frac{\alpha^{2}}{\left(7 \gamma^{2}+10 \gamma-29\right)^{2}(1+\gamma)\left(17 \gamma^{2}+13 \gamma-40\right)^{2}}\right)
\end{aligned}
$$


From the graph below we can see that the merged firm, solid line, has an incentive to merge for $\gamma \leq 0.16$ and $\gamma \geq 0.32$ while firm three, dotted line, will have an incentive to merge for $\gamma \leq 0.33$ and $\gamma \geq 0.58$

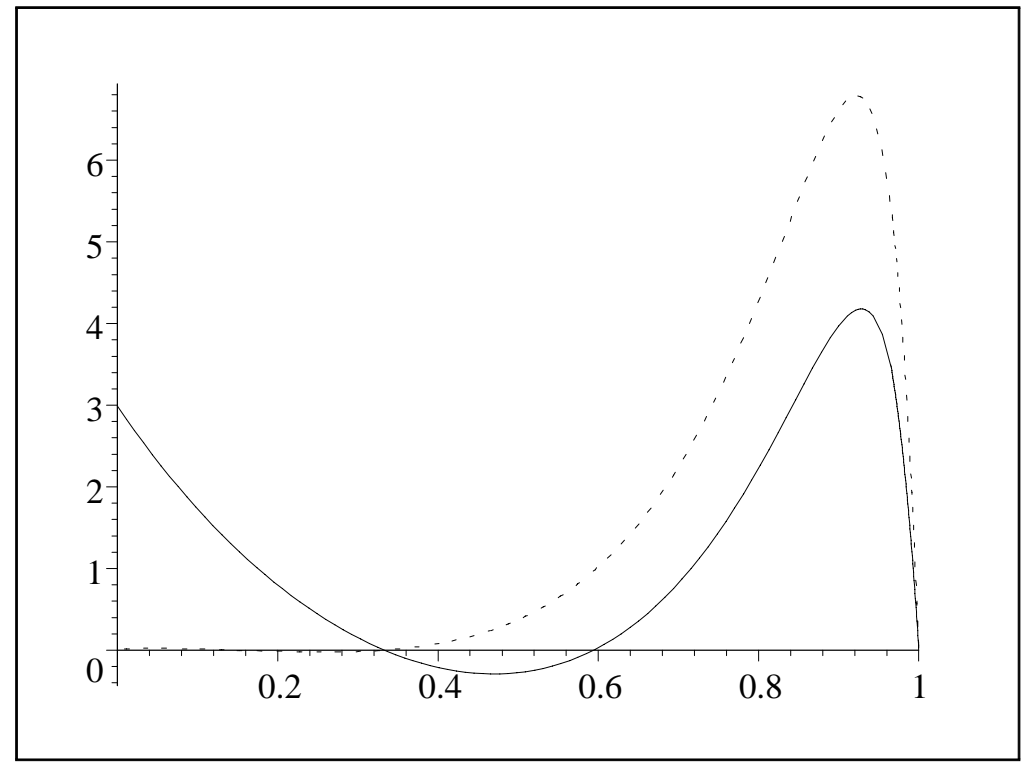

Profit Incentives

\section{Proof of Proposition 5}

From (18) and (3) we obtain the pre-merger consumer surplus for the $B_{1}$ market:

$$
U_{B}^{\text {premerg }}=\alpha^{2} \frac{\left(\gamma^{2}-2 \gamma-11\right)^{2}}{(\gamma+1)\left(7 \gamma^{2}+10 \gamma-29\right)^{2}}+m
$$

and similarly for $A_{1}$ from (4) we obtain the pre-merger consumer surplus for the A market:

$$
U_{A}^{\text {premerg }}=-\alpha^{2} \frac{3 \gamma^{4}-4 \gamma^{3}+18 \gamma^{2}-36 \gamma-125}{(\gamma+1)\left(7 \gamma^{2}+10 \gamma-29\right)^{2}}+m
$$

For the post-merger consumer surpluses we need to consider the two cases with internal and corner equilibrium prices. From (23) and (3) we obtain the post-merger consumer surplus for the $B_{1}$ market when $\gamma \lesssim 0.965$

$$
U_{B}^{\text {postmergint }}=-\frac{1}{8} \alpha^{2} \frac{76 \gamma^{4}-623 \gamma^{3}+585 \gamma^{2}+2079 \gamma-2405}{(1+\gamma)\left(8 \gamma^{2}+27 \gamma-41\right)^{2}}+m
$$

and from (23) and (4) we obtain the post-merger consumer surplus for the $A_{1}$ market when $\gamma \lesssim 0.965$

$$
U_{A}^{\text {postmeint }}=\frac{1}{8} \alpha^{2} \frac{60 \gamma^{4}+619 \gamma^{3}-757 \gamma^{2}-2075 \gamma+2441}{(\gamma+1)\left(8 \gamma^{2}+27 \gamma-41\right)^{2}}+m
$$


Finally from (25) and (3) we obtain the post-merger consumer surplus for the $B_{1}$ market when $\gamma \gtrsim 0.965$

$$
U_{B}^{\text {postmecorner }}=\frac{1}{2}\left(5 \gamma^{2}-2 \gamma-23\right)^{2} \frac{\alpha^{2}}{\left(-40-27 \gamma+30 \gamma^{2}+17 \gamma^{3}\right)^{2}}+m
$$

and from (25) and (4) we obtain the post-merger consumer surplus for the $A_{1}$ market when $\gamma \gtrsim 0.965$

$$
U_{A}^{\text {postmecorner }}=-\frac{1}{2} \alpha^{2} \frac{100 \gamma^{5}+155 \gamma^{4}-420 \gamma^{3}-182 \gamma^{2}+632 \gamma-685}{\left(-40-27 \gamma+30 \gamma^{2}+17 \gamma^{3}\right)\left(17 \gamma^{2}+13 \gamma-40\right)(\gamma+1)}+m
$$

Now subtracting (38) from (40)and from (42) we obtain the utility surplus for the $A_{1}$ market

$$
\begin{aligned}
& \Delta U_{A}= \\
& \left\{\begin{array}{cc}
\frac{1}{8} \alpha^{2} \frac{\left(\begin{array}{c}
-1430983 \gamma-314521 \gamma^{4}+1110697 \gamma^{3}+ \\
626613 \gamma^{2}++4476 \gamma^{8}+47051 \gamma^{7}+ \\
28351 \gamma^{6}-443565 \gamma^{5}+371881
\end{array}\right)}{\left(\begin{array}{c}
(\gamma+1)\left(8 \gamma^{2}+27 \gamma-41\right)^{2}\left(7 \gamma^{2}+10 \gamma-29\right)^{2} \\
-885042 \gamma^{2}+1184012 \gamma-171262 \gamma^{6}+ \\
258846 \gamma^{5}+688580 \gamma^{4}-892194 \gamma^{3}+ \\
3166 \gamma^{9}-29542 \gamma^{7}+19521 \gamma^{8}-176085
\end{array}\right)} & \\
-\frac{1}{2} \alpha^{2} \frac{}{\left(-40-27 \gamma+30 \gamma^{2}+17 \gamma^{3}\right)\left(17 \gamma^{2}+13 \gamma-40\right)(\gamma+1)\left(7 \gamma^{2}+10 \gamma-29\right)^{2}} & \gamma>0.965
\end{array}\right.
\end{aligned}
$$

and subtracting (37) from (39) and from(41) we obtain the utility surplus for the $B_{1}$ market

$$
\begin{aligned}
& \Delta U_{B}= \\
& \left\{\begin{array}{c}
-1010485 \gamma^{3}+486173 \gamma^{4}+ \\
-\frac{1}{8} \alpha^{2} \frac{\left(\begin{array}{c}
1413467 \gamma-28207 \gamma^{7}+2188 \gamma^{8}+ \\
342025 \gamma^{5}-78955 \gamma^{6}-341605-784601 \gamma^{2}
\end{array}\right)}{(1+\gamma)\left(8 \gamma^{2}+27 \gamma-41\right)^{2}\left(7 \gamma^{2}+10 \gamma-29\right)^{2}} \\
-\frac{1}{2}\left(\begin{array}{c}
578 \gamma^{9}-2075 \gamma^{8}-20270 \gamma^{7}+ \\
22170 \gamma^{6}+215710 \gamma^{5}+49444 \gamma^{4}- \\
570714 \gamma^{3}-142922 \gamma^{2}+505768 \gamma-57689
\end{array}\right) * 0.965 \\
\left(\frac{\alpha^{2}}{\left(7 \gamma^{2}+10 \gamma-29\right)^{2}(\gamma+1)\left(17 \gamma^{2}+13 \gamma-40\right)\left(-40-27 \gamma+30 \gamma^{2}+17 \gamma^{3}\right)}\right)
\end{array}\right.
\end{aligned}
$$

and finally adding (43) and (44) we obtain the merger total effect on consumer surplus. 


$$
\begin{aligned}
& \Delta U= \\
& \left\{\begin{array}{c}
\frac{1}{4} \alpha^{2} \frac{\left(\begin{array}{c}
1060591 \gamma^{3}-400347 \gamma^{4}-1422225 \gamma+37629 \gamma^{7}+ \\
1144 \gamma^{8}-392795 \gamma^{5}+53653 \gamma^{6}+356743+705607 \gamma^{2}
\end{array}\right)}{(1+\gamma)\left(8 \gamma^{2}+27 \gamma-41\right)^{2}\left(7 \gamma^{2}+10 \gamma-29\right)^{2}} \\
-\alpha^{2} \frac{\left(\begin{array}{c}
-513982 \gamma^{2}+844890 \gamma-74546 \gamma^{6}+237278 \gamma^{5}+ \\
369012 \gamma^{4}-731454 \gamma^{3}+1872 \gamma^{9}- \\
24906 \gamma^{7}+8723 \gamma^{8}-116887
\end{array}\right)}{\left(7 \gamma^{2}+10 \gamma-29\right)^{2}(\gamma+1)\left(17 \gamma^{2}+13 \gamma-40\right)\left(-40-27 \gamma+30 \gamma^{2}+17 \gamma^{3}\right)} \\
\gamma>0.965
\end{array}\right.
\end{aligned}
$$

Finally from the profits differentials $(33),(34),(35)$ and (36)we obtain the total profits differential:

$$
\begin{aligned}
& \Delta \Pi= \\
& \frac{1}{2} \alpha^{2} \frac{\left(\begin{array}{c}
9624 \gamma^{8}+52043 \gamma^{7}+49419 \gamma^{6}- \\
130309 \gamma^{5}-257309 \gamma^{4}+162713 \gamma^{3}+ \\
309289 \gamma^{2}-238047 \gamma+42577
\end{array}\right)}{\left(7 \gamma^{2}+10 \gamma-29\right)^{2}(1+\gamma)\left(8 \gamma^{2}+27 \gamma-41\right)^{2}} \quad \gamma<0.965 \\
& 2 \alpha^{2} \frac{\left(\begin{array}{c}
25772 \gamma^{7}+18385 \gamma^{8}-42836 \gamma^{6}+ \\
15314 \gamma^{5}+81078 \gamma^{4}-425348 \gamma^{3}- \\
20340 \gamma^{2}+709951 \gamma-363967+1991 \gamma^{9}
\end{array}\right)}{\left(17 \gamma^{2}+13 \gamma-40\right)^{2}(1+\gamma)^{2}\left(7 \gamma^{2}+10 \gamma-29\right)^{2}} \quad \gamma \geq 0.965
\end{aligned}
$$

Total Welfare is now given by the sum of total profits (46) and total utility (45) differentials

$$
\begin{aligned}
& \Delta W=
\end{aligned}
$$

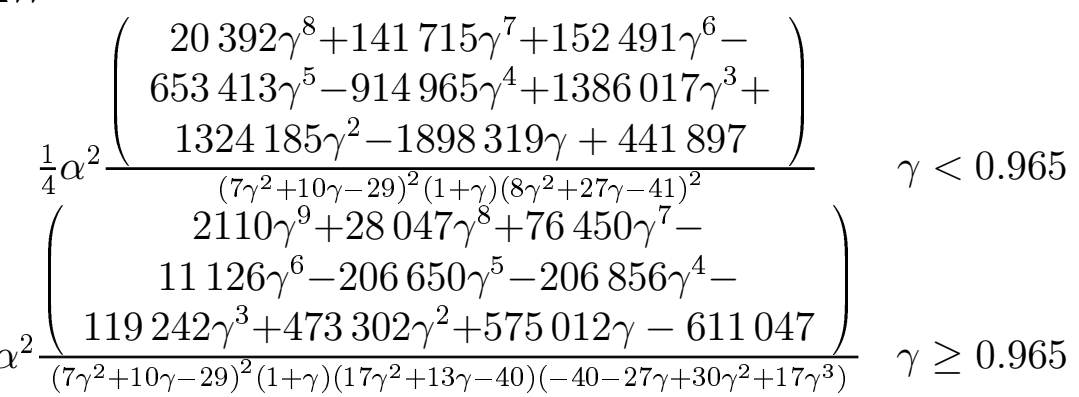

The properties of these functions prove the proposition.

\section{References}

[1] Armstrong, M.(1998). "Network Interconnection in Telecommunications", The Economic Journal, vol 108, pp. 545-564. 
[2] Armstrong, M.(2002). " The Theory of Access Pricing and Interconnection" in Handbook of Telecommunications Economics Eds M. Cave, Majumdar, S. K., and Vogelsang, A.

[3] Armstrong, M., Doyle, C. and Vickers, J. (1996) "The Access Pricing Problem: a synthesis", Journal of Industrial Economics, vol.44 pp 131-150.

[4] Economides, N. (1998), "The Telecommunications Act of 1996 and its Impact," Discussion Paper EC-98-08, Stern School of Business, N.Y.U., forthcoming, Japan and the World Economy.

[5] Economides, N. (1998), "The Incentive for non-price discrimination by an input monopolist". International Journal of Industrial Organization, vol.16, pp. 271-284.

[6] Economides, N., and Woroch, G., (1992), "Benefits and Pitfalls of Network Interconnection," Discussion Paper no. EC-92-31, Stern School of Business.

[7] A. Estache, and Valletti, T. (1999),"The theory of access pricing: an overview for infrastructure regulators", Discussion Paper 2133, CEPR, and Policy Research Working Paper 2097, The World Bank.

[8] Gibbens, R. at http://www.statslab.cam.ac.uk/ richard/PRICE/pricinginternet/

[9] Giovannetti, E. (2002), "Interconnection, Differentiation and Bottlenecks in the Internet".Information Economics and Policy. vol 14 n. 3 pp 385-404. September

[10] Laffont, J.-J.,and Tirole, J. (2000), Competition in Telecommunications Cambridge, Mass.; London: MIT Press

[11] Laffont, J.-J., Rey, P. and Tirole, J. (1998) Network Competition: I.Overview and nondiscriminatory pricing", Rand Journal of Economics, Vol 29, 1. pp 1-37.

[12] MacKie-Mason and H. R. Varian,(1995) "Pricing the Internet," in Public Access to the Internet, B. Kahin and J. Keller, eds., MIT Press, 1995, pp. 269-314.

[13] Shenker, S., D. Clark, D. Estrin, and S. Herzog, (1996); "Pricing in computer networks: reshaping the research agenda," Telecommunications Policy, 20, pp.183-201. 